Research Article

\title{
The Research on the Effect of Humidity on the Rheological Model of Swelling Rock
}

\author{
Ming Ji ${ }^{1}{ }^{1}$ and Yi-Dong Zhang ${ }^{2}$ \\ ${ }^{1}$ Key Laboratory of Deep Coal Resource Mining, Ministry of Education of China and China University of Mining \& Technology, \\ Xuzhou 221116, China \\ ${ }^{2}$ State Key Laboratory of Coal Resources and Mine Safety, China University of Mining \& Technology, Xuzhou 221116, China \\ Correspondence should be addressed to Ming Ji; jiming@cumt.edu.cn
}

Received 22 June 2020; Revised 10 July 2020; Accepted 13 July 2020; Published 14 August 2020

Academic Editor: Hailing Kong

Copyright (c) 2020 Ming Ji and Yi-Dong Zhang. This is an open access article distributed under the Creative Commons Attribution License, which permits unrestricted use, distribution, and reproduction in any medium, provided the original work is properly cited.

\begin{abstract}
The research on the rheological mechanical behaviour of swelling rock in the condition of humidity field has a significant meaning for large rock engineering, such as deep coal mining, tunnelling, and slope engineering. Based on the Nishihara model, considering on the effect of humidity factor on swelling rock, the rheological elements are established and the effects of humidity on the damage of rock, the viscosity, and the elasticity are introduced. Moreover, the viscoelastic plasticity constitutive equations are established under the coupling of swelling rock stress and humidity. Afterward, considering the effects of humidity on the damage, elasticity, and viscosity, the creeping, unloading, and relaxation equations have been deduced. From this research, under the state of low stress $\left(\sigma_{3 P}<\sigma_{S}\right)$, the Nishihara model which considers the effect of humidity shows the properties of stable creeping. In addition, the unloading curves contain instantaneous elasticity, elastic after effect, and the viscosity flow induced by the humidity. However, under the state of high stress $\left(\sigma_{3 P} \geq \sigma_{S}\right)$, it is an unstable creeping model. The unloading curves include instantaneous elasticity, elastic after effect, and the viscosity flow induced by the stress and the humidity. This model reflects the rheological properties of swelling rock comprehensively, and it can be used for rock rheological analysis.
\end{abstract}

\section{Introduction}

The research on rock rheological model is an important component of the research on rock rheological mechanical theories. Moreover, it is also one of the hot and difficult topics in the field of rock mechanics. Currently, the rheological models of rock mainly have the empirical model, the component composition model, and the damage and fracture rheological model. There are plenty of studies carried out in this field in the world. For example, Xu and Xia [1] used logarithmic empirical formulas to fit creeping test results of Granite. Zhang et al. [2] performed the uniaxial compression creeping test for Gabbro rock; they mentioned the empirical formulas about the creeping law. Zhang et al. [3] used aging theory and power functions to fund nonlinear creeping equations of Mudstone. Based on traditional linear viscoelastic models, Jin and Kui-ying [4] mentioned the nonlinear viscoelastic model. Yuan-jiang [5] used the creeping body and the fractured plastic body; these two kinds of nonlinear components found a new complex rheological model to describe soft rock. Based on the creeping test results of rock, Zhi-chun et al. [6] and Xie-xing and Chen [7] pointed out the parameters of creeping modulus and the damage equations of rock creeping which can describe damage history. Yan-yi [8] studied on the process of damage rheological fractures of jointed rock; then, he mentioned the laws of delay crack initiation and expansion and instability of rock joints under constant loading; he established the damage evolution equations of jointed rock and viscoelastic plasticity constitutive equations of damage evolution coupling. Guan et al. [9] supplied a new rheological model and applied it in mountain tunnelling. Zhang and Yang [10] suggested a nonlinear unloading rheological constitutive model based on the fractional-order 
derivative. Zhao et al. [11] examined the creep properties of limestone under unloading triaxial creep test. Nedjar and Roy [12] suggested a creep mechanical model which could describe the whole creep process of granite with different temperatures. Brotons et al. [13] studied the mechanical characteristics of limestone in a 96 hour uniaxial compressive creep test and proposed a theological model and a creep function to study the creep behaviour of rock dependent on time. Cao et al. [14] defined a new nonlinear damage creep constitutive model of high-stress soft rock with improved Burgers model, Hooke model, and St. Venant model based on the nonlinear damage creep characteristics of rock and damage variable. Lu et al. [15] proposed a timedependent model to simulate the creep aging damage, deformation, and fracture behaviour of brittle rock. Firme et al. [16] studied the elastoplastic creep characteristics of soft rock and provided an accurate explanation. Fahimifar et al. [17] modified a viscoplastic model to account for the creep deformation of rocks associated with the wide range of stress levels and implemented the model in numerical finite difference code (FLAC). Bazhin and Murashkin [18] studied the creep deformation and the stress relaxation characteristics of the material with loading and unloading condition under hydrostatic pressure and established a nonlinear rheological model.

Based on the Nishihara model in component composition model, this paper introduced the rheological components, which consider the humidity expansion effect, to establish the swelling rock viscoelastic model that considers on the humidity, the damage, and the viscosity. The research result of this paper is a useful supplement for the theory research on rock engineering.

\section{Free Expansion Experiment of Rock}

The rock sample which was obtained from the roof of a coal mine is mudstone, which will expand but cannot disintegrate, whose dry density is $2.74 \mathrm{~g} / \mathrm{cm}^{3}$.

Shaping the rock sample into a rectangle shape with length, width, and height as 5,5 , and $10 \mathrm{~cm}$. The processed sample should be heated to $105^{\circ} \mathrm{C}$ for $24 \mathrm{~h}$ in the oven to remove water completely; then, the water content of rock is 0 . In the experiment, length is tested by a vernier caliper whose range is $13.5 \mathrm{~cm}$ and precision is $0.02 \mathrm{~mm}$; height is tested by an electronic balance JM10002 whose range is $1000 \mathrm{~g}$ and precision is $0.01 \mathrm{~g}$. Taking the dry rock sample out and putting into a desiccator for cooling, then measure and record length $(x)$, width $(y)$, height $(z)$, and mass $(m)$ of the dry sample. Lay the test area of the dry sample and measure its length, width, and height with the method getting the average value by multiple measuring.

The expanding experiment was processing in the environment of $20^{\circ} \mathrm{C}$. Put the sample into water and ensure it soaks completely and expands freely; then, take the sample out at an interval, dry the surface of it with absorbent paper, and measure the data: length, width, height, and mass. The standard to judge the sample whether to be water saturating is the mass of the rock increases less than $0.1 \mathrm{~g} / \mathrm{h}$ one time.
Record the experimental data of every time points; the result of expansion strain and the water content is shown in Table 1:

\section{The Relationship between Expansion Strain and Water Content}

In [19-21], the concept of humidity stress field is shown, which held the point that humidity diffusion in the swelling rock has coupling relationship with water content, water absorption force, and volume deformation. Let the geotechnical expand freely, and it will bring a strain component as follows:

$$
\varepsilon_{i j}=\alpha \delta_{i j} \Delta w
$$

Among the formula, $\alpha$ is linear expansion coefficient, $\delta_{i j}$ is a symbol of Kronecker, and $\Delta w$ is a humidity changing value.

Figure 1 shows the relationship between expansion strain and volume water content and mass water content of lime mudstone in 3 directions at length, width, and height.

From Figure 1 we know that the free expansion strain has proper linear relationship with both volume water content and mass water content of the rock sample in 3 directions at length, width, and height, whose result is fit for the humidity stress field concept in [19-21]. We found the expansion coefficient of volume water content as $0.01421,0.01603$, and 0.01782 and mass water content as $0.03852,0.04345$, and 0.04831 , respectively, in three directions at length, width, and height through function fitting.

The anisotropy of the experimental rock sample leads to the difference of expansion coefficient in three directions at length, width, and height, but the difference is very small from the data, so the rock sample can be approximately regarded as isotropic rock mass. So, the relationship between free expansion strain and water content of lime mudstone can be shown as follows:

$$
\begin{aligned}
& \varepsilon=K_{m} w_{m}, \\
& \varepsilon=K_{v} w_{v} .
\end{aligned}
$$

Among the formula, $w_{m}$ and $w_{v}$, respectively, show the mass water content and volume water content and $K_{m}$ and $K_{v}$ show the corresponding expansion coefficients.

\section{The Constitutive Model}

The fundamental components of the conventional rheological model only considered the effect of the load; however, the effect of humidity factor also has obvious impact on the mechanical properties of swelling rock. Considering the effect of humidity factor and combining with the humidity stress field theory, the following hypotheses have been mentioned (Table 2).

The Nishihara model is series connected by the Hooker model, the Kelvin model, and the ideal viscoplastic model. In this paper, based on the Nishihara model, the humidity effect is introduced; then, as shown in Figure 2, the Nishihara 
TABLE 1: Experiment results.

\begin{tabular}{lccccc}
\hline$T(h)$ & \multicolumn{3}{c}{ Expansion strain $\left(10^{-2}\right)$} & Mass content & Volume content \\
\hline 0 & $\varepsilon_{x}$ & $\varepsilon_{y}$ & $\varepsilon_{z}$ & 0 & 0 \\
0.2 & 0 & 0 & 0 & 0.031969 & 0.08735 \\
0.4 & 0.0799 & 0.1201 & 0.1406 & 0.042694 & 0.116374 \\
0.8 & 0.1599 & 0.2002 & 0.2209 & 0.055719 & 0.151665 \\
1.2 & 0.1998 & 0.2402 & 0.2812 & 0.064906 & 0.210701 \\
2 & 0.2398 & 0.2802 & 0.3213 & 0.077594 & 0.230556 \\
4 & 0.2798 & 0.3203 & 0.3615 & 0.085007 & 0.247188 \\
10 & 0.3197 & 0.3603 & 0.4017 & 0.091249 & 0.100289 \\
24 & 0.3597 & 0.4003 & 0.4419 & 0.10697 & 0.289083 \\
48 & 0.3997 & 0.4404 & 0.482 & 0.111995 & 0.302603 \\
72 & 0.4396 & 0.4804 & 0.5222 & 0.111995 & 0.302603 \\
96 & 0.4396 & 0.4804 & 0.5423 & 0.5423 & \\
\hline
\end{tabular}

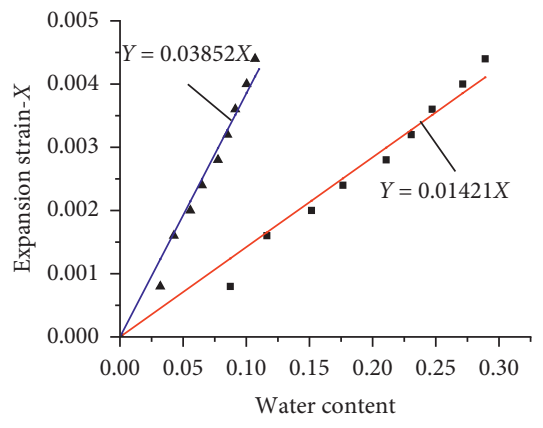

- Volume water content

\ Mass water content

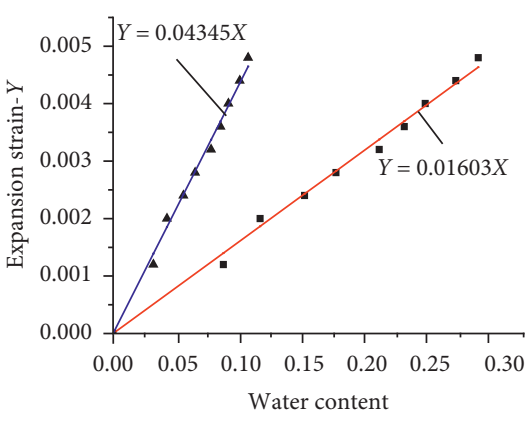

- Volume water content

- Mass water content

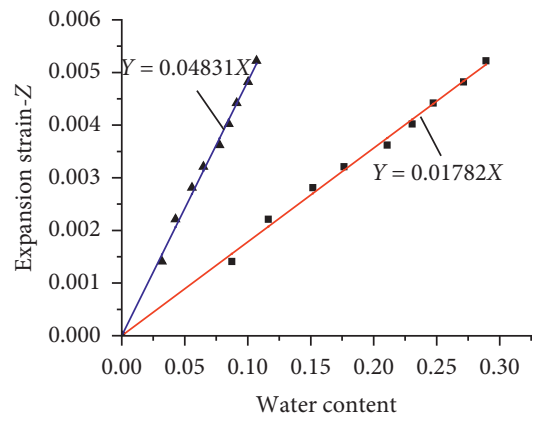

- Volume water content

- Mass water content

FIGURE 1: Relationship between expansion strain and water content.

TABLe 2: Rheological elements in the humidity field.

\begin{tabular}{ll}
\hline $\begin{array}{l}\text { Fundamental } \\
\text { components }\end{array}$ & \multicolumn{2}{c}{ Symbols } \\
\hline Hooker model & $\frac{\sigma}{\Delta w} \alpha$
\end{tabular}

Plastic mode

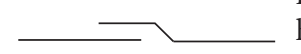

$\sigma$ Is the stress; $E$ and $a$ are the elasticity coefficient and the humidity expansion coefficient of Hooker model, respectively; $\Delta w$ is the humidity difference of before and after two states; moreover, assume that the changes of the humidity have no effect on the Hooker law of the elastic model

When $\sigma<\sigma_{s}$, there is no strain changes; when $\sigma>\sigma_{s}$, the plastic model yield; $\sigma_{s}$ is the yield limit of the material; when the stress meets the yield limit, the yield continues, no-slip in friction surface and have no relationship with the time; in the coupling of humidity and stress, the yield stress of the material is $\sigma_{s}(w)$ and the yield surface is $f\left(\sigma_{s}, w\right)$, both of them have the relationships with the humidity; moreover, assume that the yield stress is a given constant

Newton
model

The stress and strain obey to sticky laws in the Newton model; there is a proportional relationship between the stress and the strain rate; the formulation is $\sigma=\eta \dot{\varepsilon}, \eta$ is the sticky coefficient; under the coupling of the humidity and the stress, the sticky coefficient is expressed by $\eta(w)$ and it has the relationship with the humidity; moreover, assume that the sticky coefficient is a given constant

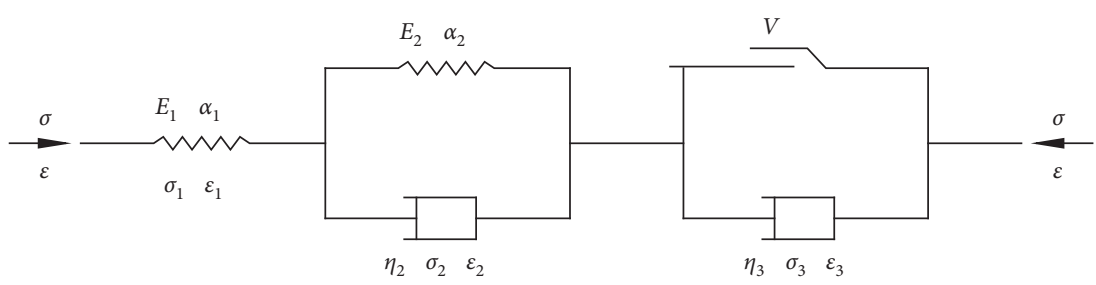

FIgUre 2: The Nishihara model under the humidity effect. 
model, which considers the effect of the humidity, has been found.

Under the coupling of the humidity-stress, the stressstrain relationships in this model can be shown as follows:

$$
\left\{\begin{array}{l}
\sigma=\sigma_{1}=\sigma_{2}=\sigma_{3}, \\
\varepsilon=\varepsilon_{1}+\varepsilon_{2}+\varepsilon_{3} .
\end{array}\right.
$$

In these formulas, $\sigma_{1}=E_{1} \varepsilon_{1}-E_{1} \alpha_{1} \Delta w$,

$$
\begin{aligned}
& \left\{\begin{array}{l}
\sigma_{2}=\sigma_{2 E}+\sigma_{2 \eta}, \\
\sigma_{2 E}=E_{2} \varepsilon_{2 E}-E_{2} \alpha_{2} \Delta w, \\
\sigma_{2 \eta}=\eta_{2}(w) \dot{\varepsilon}_{2 \eta},
\end{array}\right. \\
& \left\{\begin{array}{l}
\sigma_{3}=\sigma_{3 P}+\sigma_{3 \eta}, \\
\sigma_{3 P}= \begin{cases}0, & \sigma_{3 P}<\sigma_{s}, \\
\sigma_{s}, & \sigma_{3 P} \geq \sigma_{s},\end{cases} \\
\sigma_{3 \eta}=\eta_{3}(w) \dot{\varepsilon}_{3 \eta},
\end{array}\right. \\
& \left\{\begin{array}{l}
\varepsilon_{2}=\varepsilon_{2 E}=\varepsilon_{2 \eta}, \\
\varepsilon_{3 P}= \begin{cases}0, & \sigma_{3 P}<\sigma_{s} \\
\varepsilon_{3}, & \sigma_{3 P} \geq \sigma_{s}\end{cases} \\
\varepsilon_{3}=\varepsilon_{3 P}=\varepsilon_{3 \eta} .
\end{array}\right.
\end{aligned}
$$

In these formulas, $\sigma$ is the total stress of this model; $\varepsilon$ is the total strain of this model; $\Delta w$ is the amount of the humidity changes between two states; $\sigma_{2}$ and $\varepsilon_{2}$ are the total stress and the total strain in the Kelvin model, respectively; $\sigma_{3}$ and $\varepsilon_{3}$ are the total stress and the total strain in the ideal viscoplastic model, respectively; $\sigma_{1}, E_{1}, \varepsilon_{1}$, and $\alpha_{1}$ are the stress, the elastic module, the strain, and the wet expansion coefficient in the Hooker model respectively; $\sigma_{2 \mathrm{E}}, E_{2}, \varepsilon_{2 \mathrm{E}}$, and $\alpha_{2}$ are the stress, the elastic module, the strain, and the wet expansion coefficient in the Kelvin model, respectively; $\sigma_{2 \eta}$, $\varepsilon_{2 \eta}$, and $\eta_{2}(w)$ are the stress of the viscous model, the strain, and the wet viscosity coefficient of the Kelvin model, respectively; $\sigma_{3 \eta}, \varepsilon_{3 \eta}$, and $\eta_{3}(w)$ are the stress of the viscous model, the strain, and the wet viscosity coefficient of the ideal viscoplastic model, respectively; $\sigma_{3 \mathrm{P}}, \varepsilon_{3}$ P and $\sigma_{3 \mathrm{~S}}$ are the stress of elastic components, the strain, and the yield limit of the ideal viscoplastic model, respectively.

Under the state of low stress $\left(\sigma_{3 P}<\sigma_{S}\right)$, the ideal viscoplastic model has no effect and the model is the generalized Kelvin model, as shown in Figure 3.

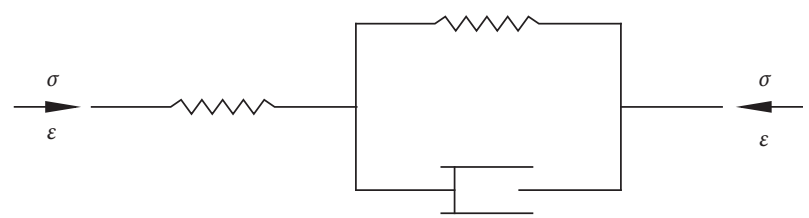

FIgURE 3: The generalized Kelvin model with the humidity effect.

In Figure 2, $\sigma_{1}=\sigma_{2}=\sigma, \varepsilon_{1}+\varepsilon_{2}=\varepsilon$, and $\dot{\varepsilon}_{1}+\dot{\varepsilon}_{2}=\dot{\varepsilon}$.

For the Hooker model, $\sigma=E_{1} \dot{\varepsilon}_{1}-E_{1} \alpha_{1} \Delta \dot{w}$ and $\dot{\sigma}=E_{1} \dot{\varepsilon}_{1}-E_{1} \alpha_{1} \Delta \dot{w}$.

For the Kelvin model, $\sigma=E_{2} \varepsilon_{2}-E_{2} \alpha_{2} \Delta w+\eta_{2}(w) \dot{\varepsilon}_{2}$.

Thus, $\sigma=E_{2}\left(\varepsilon-\varepsilon_{1}\right)-E_{2} \alpha_{2} \Delta w+\eta_{2}(w)\left(\dot{\varepsilon}-\dot{\varepsilon}_{1}\right)$.

Then, the constitutive equation, which considers the effect of the humidity on the elasticity, can be deduced:

$$
\begin{aligned}
& \frac{\eta_{2}(w)}{E_{1}} \dot{\sigma}+\left(1+\frac{E_{2}}{E_{1}}\right) \sigma+\eta_{2}(w) \alpha_{1} \Delta \dot{w}+E_{2}\left(\alpha_{1}+\alpha_{2}\right) \Delta w \\
& =\eta_{2}(w) \dot{\varepsilon}+E_{2} \varepsilon .
\end{aligned}
$$

Under high-stress state $\left(\sigma_{3 P} \geq \sigma_{S}\right)$, as shown in Figure 1, this model has three components. Moreover, the constitutive equation can be deduced with the similar process:

$$
\begin{aligned}
\ddot{\sigma} & +\left(\frac{E_{1}}{\eta_{2}(w)}+\frac{E_{1}}{\eta_{3}(w)}+\frac{E_{2}}{\eta_{2}(w)}\right) \dot{\sigma}+\frac{E_{1} E_{2}}{\eta_{2}(w) \eta_{3}(w)}\left(\sigma-\sigma_{s}\right) \\
& +E_{1} \alpha \alpha_{1} \Delta \ddot{w}+\frac{E_{1} E_{2}\left(\alpha_{1}+\alpha_{2}\right)}{\eta_{2}(w)} \Delta \dot{w}=E_{1} \ddot{\varepsilon}+\frac{E_{1} E_{2}}{\eta_{2}(w)} \dot{\varepsilon} .
\end{aligned}
$$

The humidity and the humidity content have dramatic effects on the creeping properties of the rock. Generally, the rock strength will decrease with the increase of the humidity; however, the rate of rock creeping will increase. According to [22], the viscosity coefficient will decrease with the increase of the humidity; moreover, it is almost a linear change. If $\xi_{2}$ and $\xi_{3}$ are presumed as the corresponding viscosity attenuation coefficient, $\eta_{2}^{\prime}(w)=\eta_{2}(w)-\xi_{2} \Delta w$ and $\eta_{3}^{\prime}(w)=\eta_{3}(w)-\xi_{3} \Delta w$. Then, the constitutive relationship is

$$
\left\{\begin{array}{c}
\frac{\eta_{2}(w)-\xi_{2} \Delta w}{E_{1}} \dot{\sigma}+\left(1+\frac{E_{2}}{E_{1}}\right) \sigma+\left[\eta_{2}(w)-\xi_{2} \Delta w\right] \alpha_{1} \Delta \dot{w}+E_{2}\left(\alpha_{1}+\alpha_{2}\right) \Delta w=\left[\eta_{2}(w)-\xi_{2} \Delta w\right] \dot{\varepsilon}+E_{2} \varepsilon, \quad \sigma_{3 P}<\sigma_{s}, \\
\ddot{\sigma}+\left(\frac{E_{1}}{\eta_{2}(w)-\xi_{2} \Delta w}+\frac{E_{1}}{\eta_{3}(w)-\xi_{3} \Delta w}+\frac{E_{2}}{\eta_{2}(w)-\xi_{2} \Delta w}\right) \dot{\sigma}+\frac{E_{1} E_{2}}{\left[\eta_{2}(w)-\xi_{2} \Delta w\right]\left[\eta_{3}(w)-\xi_{3} \Delta w\right]}\left(\sigma-\sigma_{s}\right) \\
+E_{1} \alpha_{1} \Delta \ddot{w}+\frac{E_{1} E_{2}\left(\alpha_{1}+\alpha_{2}\right)}{\eta_{2}(w)-\xi_{2} \Delta w} \Delta \dot{w}=E_{1} \ddot{\varepsilon}+\frac{E_{1} E_{2}}{\eta_{2}(w)-\xi_{2} \Delta w} \dot{\varepsilon}, \quad \sigma_{3 P} \geq \sigma_{s} .
\end{array}\right.
$$


Large amounts of experiments have identified that the properties of swelling rock will be reduced after it is contacted with water; moreover, the elastic modulus will also be decreased with the increase of the humidity [23]. Thus, introducing two damage factors $\left(D_{1}\right.$ and $\left.D_{2}\right)$ for the Hooker element, then $E_{1}^{\prime}=E_{1}\left(1-D_{1}\right)$ and $E_{2}^{\prime}=E_{2}\left(1-D_{2}\right)$, and the constitutive equations are

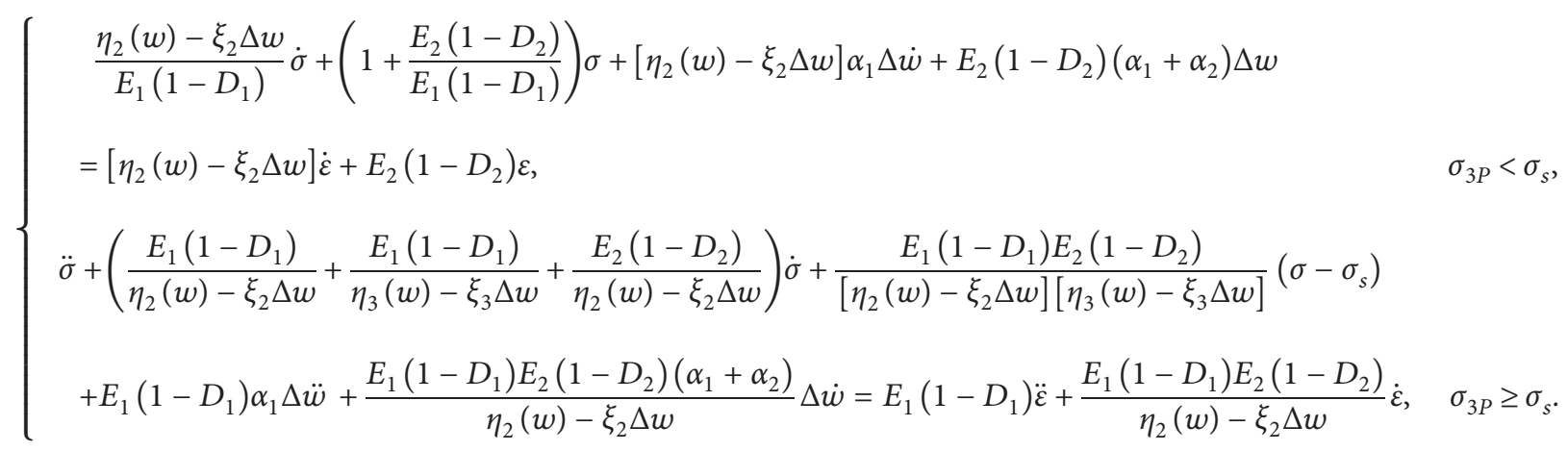

4.1. Creeping Equations. Assuming that the humidity is constant after the absorbent of the rock; then, $\Delta \dot{w}=0$.

Under the state of low stress $\left(\sigma_{3 P}<\sigma_{S}\right)$, it has a constant value, $\dot{\sigma}=0$. Then, the model is the generalized Kelvin model. For the Hooker model, when $\sigma=\sigma_{0}$, then $\varepsilon_{1}(t)=\left(\sigma_{0} / E_{1}\right)+\alpha_{1} \Delta w$. For the Kelvin model, $\sigma_{0}=E_{2} \varepsilon_{2}(t)-E_{2} \alpha_{2} \Delta w+\eta_{2}(w)\left(\mathrm{d} \varepsilon_{2}(t) / \mathrm{d} t\right) ; \quad$ then, $\varepsilon_{2}(t)=\left(\sigma_{0}+E_{2} \alpha_{2} \Delta w / E_{2}\right)+A e^{-\left(E_{2} / \eta_{2}(w)\right) t}$; in this equation, $A$ is the integration constant. When $t=0, \varepsilon_{2}=0$; then, $A=-\left(\sigma_{0}+E_{2} \alpha_{2} \Delta w / E_{2}\right)$. The creeping equation is

$$
\varepsilon(t)=\varepsilon_{1}(t)+\varepsilon_{2}(t)=\frac{\sigma_{0}}{E_{1}}+\alpha_{1} \Delta w+\frac{\sigma_{0}+E_{2} \alpha_{2} \Delta w}{E_{2}}\left(1-e^{-\left(E_{2} / \eta_{2}(w)\right) t}\right) .
$$

When $t$ tends to infinity, $\varepsilon(t)=\left(\sigma_{0} / E_{1}\right)+\alpha_{1} \Delta w+\left(\sigma_{0} / E_{2}\right)+\alpha_{2} \Delta w$ is the horizontal asymptote of the creeping equation and this model is under stable creeping.
Under the state of high stress $\left(\sigma_{3 P} \geq \sigma_{S}\right)$, the rock has plastic deformation. For the Hooker model, $\varepsilon_{1}(t)=\left(\sigma_{0} / E_{1}\right)+\alpha_{1} \Delta w$. For the Kelvin model, $\varepsilon_{2}(t)=\left(\sigma_{0}+E_{2} \alpha_{2} \Delta w / E_{2}\right)\left(1-e^{-\left(E_{2} / \eta_{2}(w)\right) t}\right)$. For the ideal viscoplastic model, $\varepsilon_{3}(t)=\left(\sigma_{0}-\sigma_{s} / \eta_{3}(w)\right) t$. The creeping equation is

$$
\begin{aligned}
\mathcal{\varepsilon}(t)= & \varepsilon_{1}(t)+\varepsilon_{2}(t)+\varepsilon_{3}(t)=\frac{\sigma_{0}}{E_{1}}+\alpha_{1} \Delta w+\frac{\sigma_{0}+E_{2} \alpha_{2} \Delta w}{E_{2}} \\
& \cdot\left(1-e^{-\left(E_{2} / \eta_{2}(w)\right) t}\right)+\frac{\sigma_{0}-\sigma_{s}}{\eta_{3}(w)} t .
\end{aligned}
$$

This model will become larger as the time goes; the strain will be reduced gradually; this model will be under unstable creeping and the creeping will be instability finally.

The creeping equation considers the effects of humidity on elasticity and viscosity as follows:

$$
\mathcal{E}(t)=\left\{\begin{array}{lr}
\frac{\sigma_{0}}{E_{1}}+\alpha_{1} \Delta w+\frac{\sigma_{0}+E_{2} \alpha_{2} \Delta w}{E_{2}}\left(1-e^{-\left(E_{2} /\left(\eta_{2}(w)-\xi_{2} \Delta w\right)\right) t}\right), & \sigma_{3 P}<\sigma_{s}, \\
\frac{\sigma_{0}}{E_{1}}+\alpha_{1} \Delta w+\frac{\sigma_{0}+E_{2} \alpha_{2} \Delta w}{E_{2}}\left(1-e^{-\left(E_{2} /\left(\eta_{2}(w)-\xi_{2} \Delta w\right)\right) t}\right)+\frac{\sigma_{0}-\sigma_{s}}{\eta_{3}(w)} t, & \sigma_{3 P} \geq \sigma_{s} .
\end{array}\right.
$$

The creeping equation considers the effects of humidity on elasticity, damage, and viscosity as follows:

$$
\varepsilon(t)= \begin{cases}\frac{\sigma_{0}}{E_{1}\left(1-D_{1}\right)}+\alpha_{1} \Delta w+\frac{\sigma_{0}+E_{2}\left(1-D_{2}\right) \alpha_{2} \Delta w}{E_{2}\left(1-D_{2}\right)}\left(1-e^{-\left(E_{2}\left(1-D_{2}\right) /\left(\eta_{2}(w)-\xi_{2} \Delta w\right)\right) t}\right), & \sigma_{3 P}<\sigma_{s}, \\ \frac{\sigma_{0}}{E_{1}\left(1-D_{1}\right)}+\alpha_{1} \Delta w+\frac{\sigma_{0}+E_{2}\left(1-D_{2}\right) \alpha_{2} \Delta w}{E_{2}\left(1-D_{2}\right)}\left(1-e^{-\left(E_{2}\left(1-D_{2}\right) /\left(\eta_{2}(w)-\xi_{2} \Delta w\right)\right) t}\right)+\frac{\sigma_{0}-\sigma_{s}}{\eta_{3}(w)-\xi_{3} \Delta w} t, & \sigma_{3 P} \geq \sigma_{s} .\end{cases}
$$


4.2. Unloading Equations. Under the state of low stress $\left(\sigma_{3 P}<\sigma_{s},\right)$, unloading at the time of $t_{1}$, the stress is zero. For the Hooker model, $\varepsilon_{1}(t)=\alpha_{1} \Delta w$.

For the Kelvin model, $\left(\mathrm{d} \varepsilon_{2}(t) / \mathrm{d} t\right)+\left(E_{2} \varepsilon_{2}(t) / \eta_{2}(w)\right)=\left(E_{2} \alpha_{2} \Delta w / \eta_{2}(w)\right) ; \quad$ then, $\varepsilon_{2}(t)=\alpha_{2} \Delta w+B e^{-\left(E_{2} / \eta_{2}(w)\right) t}$. In this equation, $\mathrm{B}$ is integration constant. A the time of $t_{1}$, $\varepsilon_{2}=\varepsilon_{21}=\left(\sigma_{0}+E_{2} \alpha_{2} \Delta w / E_{2}\right)\left(1-e^{-\left(E_{2} / \eta_{2}(w)\right) t_{1}}\right) ; \quad$ then, $B=\left(\varepsilon_{21}-\alpha_{2} \Delta w\right) e^{-\left(E_{2} / \eta_{2}(w)\right) t_{1}}$. The unloading equation is

$$
\begin{aligned}
\mathcal{E}(t)= & \varepsilon_{1}(t)+\varepsilon_{2}(t)=\left(\alpha_{1}+\alpha_{2}\right) \Delta w+\left(\varepsilon_{21}-\alpha_{2} \Delta w\right) \\
& e^{-\left(E_{2} / \eta_{2}(w)\right)\left(t_{1}-t\right)} .
\end{aligned}
$$

When $t$ tends to infinite, $\varepsilon(t)=\left(\alpha_{1}+\alpha_{2}\right) \Delta w$. Thus, because of the effect of humidity, the deformation of the model is not recovered to zero:

$$
\begin{aligned}
\mathcal{E}(t)= & \varepsilon_{1}(t)+\varepsilon_{2}(t)+\varepsilon_{3}(t)=\left(\alpha_{1}+\alpha_{2}\right) \Delta w+\left(\varepsilon_{21}-\alpha_{2} \Delta w\right) \\
& \cdot e^{-\left(E_{2} / \eta_{2}(w)\right)\left(t_{1}-t\right)}+\frac{\sigma_{0}-\sigma_{s}}{\eta_{3}(w)} t_{1} .
\end{aligned}
$$

Under the state of high stress $\left(\sigma_{3 P} \geq \sigma_{s}\right)$, for the Hooker model, $\varepsilon_{1}(t)=\alpha_{1} \Delta w$. For the Kelvin model, $\varepsilon_{2}(t)=\alpha_{2} \Delta w+\left(\varepsilon_{21}-\alpha_{2} \Delta w\right) e^{-\left(E_{2} / \eta_{2}(w)\right)\left(t_{1}-t\right)}$. For the ideal viscoplastic model, the model remains at the time of $t_{1}$ after unloading, the strain is $\varepsilon_{3}=\left(\sigma_{0}-\sigma_{s} / \eta_{3}(w)\right) t_{1}$, and the deformation is the eternal plastic deformation. The unloading equation is

$$
\begin{aligned}
\varepsilon(t)= & \varepsilon_{1}(t)+\varepsilon_{2}(t)+\varepsilon_{3}(t)=\left(\alpha_{1}+\alpha_{2}\right) \Delta w \\
& +\left(\varepsilon_{21}-\alpha_{2} \Delta w\right) e^{-\left(E_{2} / \eta_{2}(w)\right)\left(t_{1}-t\right)}+\frac{\sigma_{0}-\sigma_{s}}{\eta_{3}(w)} t_{1} .
\end{aligned}
$$

When $t$ tends to infinite, $\varepsilon(t)=\left(\alpha_{1}+\alpha_{2}\right) \Delta w+\left(\sigma_{0}-\sigma_{s} / \eta_{3}(w)\right) t_{1}$.

Considering about the effects of the humidity on the elasticity and the viscosity, the unloading equation is as follows:

$$
\mathcal{E}(t)= \begin{cases}\left(\alpha_{1}+\alpha_{2}\right) \Delta w+B e^{-\left(E_{2} /\left(\eta_{2}(w)-\xi_{2} \Delta w\right)\right) t} & \sigma_{3 P}<\sigma_{s}, \\ \left(\alpha_{1}+\alpha_{2}\right) \Delta w+B e^{-\left(E_{2} /\left(\eta_{2}(w)-\xi_{2} \Delta w\right)\right) t}+\frac{\sigma_{0}-\sigma_{s}}{\eta_{3}(w)-\xi_{3} \Delta w} t_{1}, & \sigma_{3 P} \geq \sigma_{s} .\end{cases}
$$

In this equation, $B=\left(\varepsilon_{21}-\alpha_{2} \Delta w\right) e^{-\left(E_{2} /\left(\eta_{2}(w)-\xi_{2} \Delta w\right)\right) t_{1}}$.

$$
\varepsilon(t)= \begin{cases}\left(\alpha_{1}+\alpha_{2}\right) \Delta w+B e^{-\left(E_{2}\left(1-D_{2}\right) /\left(\eta_{2}(w)-\xi_{2} \Delta w\right)\right) t,} & \sigma_{3 P}<\sigma_{s}, \\ \left(\alpha_{1}+\alpha_{2}\right) \Delta w+B e^{-\left(E_{2}\left(1-D_{2}\right) /\left(\eta_{2}(w)-\xi_{2} \Delta w\right)\right) t} \frac{\sigma_{0}-\sigma_{s}}{\eta_{3}(w)-\xi_{3} \Delta w} t_{1}, & \sigma_{3 P} \geq \sigma_{s} .\end{cases}
$$

In this equation, $B=\left(\varepsilon_{21}-\alpha_{2} \Delta w\right) e^{-\left(E_{2}\left(1-D_{2}\right) /\left(\eta_{2}(w)-\xi_{2} \Delta w\right)\right) t 1}$.

4.3. Relaxation Equations. Under the state of low stress $\left(\sigma_{3 P}<\sigma_{s},\right)$ and $\varepsilon=\varepsilon_{0}$. That is, $\dot{\varepsilon}=0$. Then, $\dot{\sigma}+\left(E_{1}+\right.$ $\left.E_{2} / \eta_{2}(w)\right) \sigma=\left(E_{1} / \eta_{2}(w)\right)\left[E_{2} \varepsilon_{0}-E_{2}\left(\alpha_{1}+\alpha_{2}\right) \Delta w\right]$, introducing $t=0$ and $\sigma=\sigma_{0}$ into it; then, $\sigma_{0}=E_{1} \varepsilon_{0}$ :

$$
\begin{aligned}
\sigma= & -\frac{E_{1}}{E_{1}+E_{2}}\left[E_{1} \varepsilon_{0}+E_{2}\left(\alpha_{1}+\alpha_{2}\right) \Delta w\right] e^{-\left(E_{1}+E_{2} / \eta_{2}(w)\right) t} \\
& +\frac{E_{1} E_{2}}{E_{1}+E_{2}}\left[\varepsilon_{0}-\left(\alpha_{1}+\alpha_{2}\right) \Delta w\right] .
\end{aligned}
$$

Considering the effects of the humidity on elasticity, damage, and viscosity, the unloading equation is as follows: 


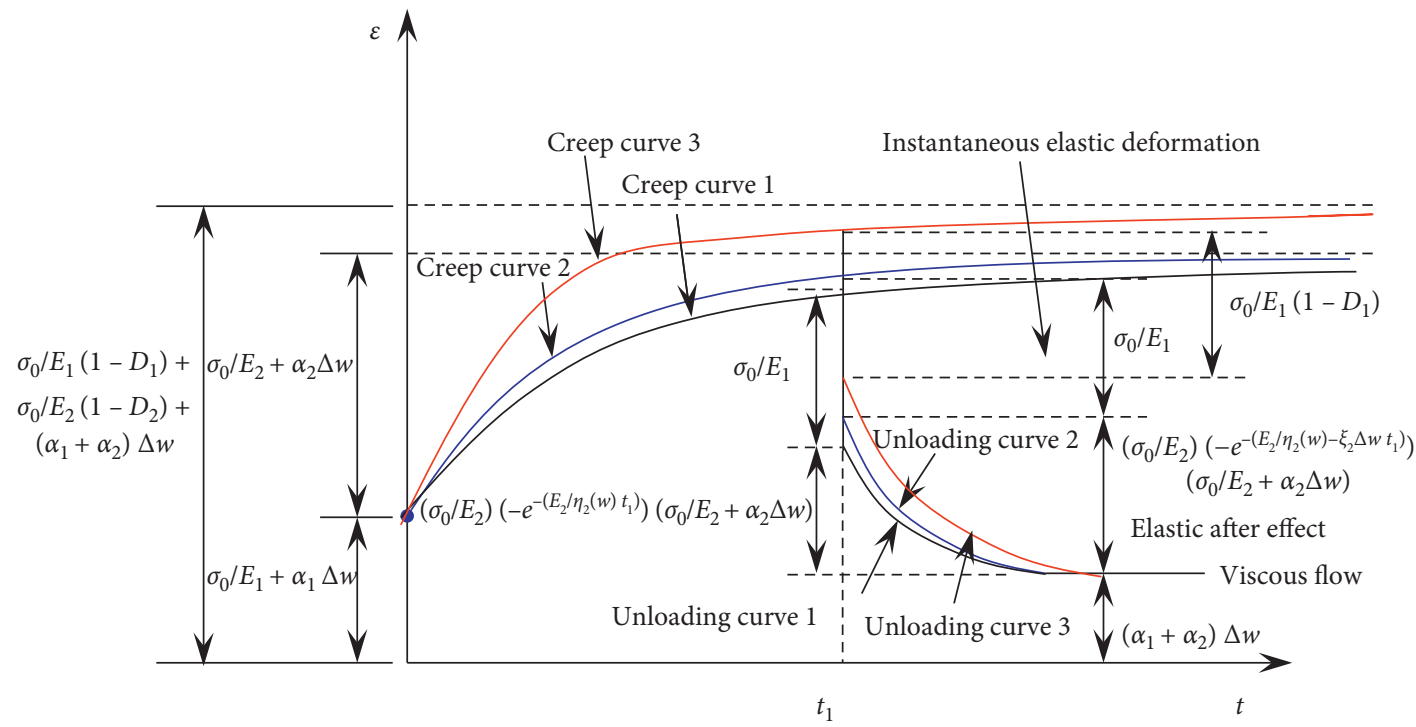

Figure 4: Model curves of creeping and unloading while $\sigma_{3 P}<\sigma_{S}$.

$$
\sigma= \begin{cases}\left\{-\frac{E_{1}}{E_{1}+E_{2}}\left[E_{1} \varepsilon_{0}+E_{2}\left(\alpha_{1}+\alpha_{2}\right) \Delta w\right]\right\} e^{-\left(E_{1}+E_{2} /\left(\eta_{2}(w)-\xi_{2} \Delta w\right)\right) t}+\frac{E_{1} E_{2}}{E_{1}+E_{2}}\left[\varepsilon_{0}-\left(\alpha_{1}+\alpha_{2}\right) \Delta w\right], & \sigma_{3 P}<\sigma_{s}, \\ \left\{-\frac{E_{1}}{E_{1}+E_{2}}\left[E_{1} \varepsilon_{0}+E_{2}\left(\alpha_{1}+\alpha_{2}\right) \Delta w\right]\right\} e^{-\left(E_{1}+E_{2} /\left(\eta_{2}(w)-\xi_{2} \Delta w\right)\right) t}+\frac{E_{1} E_{2}}{E_{1}+E_{2}}\left[\varepsilon_{0}-\left(\alpha_{1}+\alpha_{2}\right) \Delta w\right]+\sigma_{s}, & \sigma_{3 P} \geq \sigma_{s} .\end{cases}
$$

The below is the relaxation equation which considers the effects of humidity on elasticity, damage, and viscosity.

$$
\begin{aligned}
& \left\{\left\{-\frac{E_{1}\left(1-D_{1}\right)}{E_{1}\left(1-D_{1}\right)+E_{2}\left(1-D_{2}\right)}\left[E_{1}\left(1-D_{1}\right) \varepsilon_{0}+E_{2}\left(1-D_{2}\right)\left(\alpha_{1}+\alpha_{2}\right) \Delta w\right]\right\} e^{-\left(\left(E_{1}\left(1-D_{1}\right)+E_{2}\left(1-D_{2}\right)\right) /\left(\eta_{2}(w)-\xi_{2} \Delta w\right)\right) t}\right. \\
& +\frac{E_{1}\left(1-D_{1}\right) E_{2}\left(1-D_{2}\right)}{E_{1}\left(1-D_{1}\right)+E_{2}\left(1-D_{2}\right)}\left[\varepsilon_{0}-\left(\alpha_{1}+\alpha_{2}\right) \Delta w\right], \quad \sigma_{3 P}<\sigma_{s} \\
& \sigma=\{ \\
& \left\{-\frac{E_{1}\left(1-D_{1}\right)}{E_{1}\left(1-D_{1}\right)+E_{2}\left(1-D_{2}\right)}\left[E_{1}\left(1-D_{1}\right) \varepsilon_{0}+E_{2}\left(1-D_{2}\right)\left(\alpha_{1}+\alpha_{2}\right) \Delta w\right]\right\} e^{-\left(\left(E_{1}\left(1-D_{1}\right)+E_{2}\left(1-D_{2}\right)\right) /\left(\eta_{2}(w)-\xi_{2} \Delta w\right)\right) t} \\
& +\frac{E_{1}\left(1-D_{1}\right) E_{2}\left(1-D_{2}\right)}{E_{1}\left(1-D_{1}\right)+E_{2}\left(1-D_{2}\right)}\left[\varepsilon_{0}-\left(\alpha_{1}+\alpha_{2}\right) \Delta w\right]+\sigma_{s} \\
& \sigma_{3 P} \geq \sigma_{s} .
\end{aligned}
$$

Figures 4 and 5 show the creeping curves and the unloading curves of the model under two states of the stress, respectively. The creeping curve 1 and the unloading curve 1 indicate that the effect of the humidity on the elasticity is only considered on. The creeping curve 2 and the unloading curve 2 express that the effects of the humidity on the elasticity and the viscosity are considered about. The creeping curve 3 and the unloading curve 3 show that the effects of humidity on elasticity, damage, and viscosity are considered about.
According to Figures 4 and 5, under three situations, when $\sigma_{3 P}<\sigma_{S}$, the model has a relatively fast deformation at the beginning; afterward, and it tends to stable gradually. Based on the unloading curve, the model has the instantaneous elastic deformation, elastic after effect, and the viscous flow which are caused by the humidity. At the situation of $\sigma_{3 P} \geq \sigma_{S}$, it transfers to the unstable creeping. According to the unloading curve, it has the instantaneous elastic deformation, elastic after effect, and viscosity flow induced by both of humidity and stress. This model 


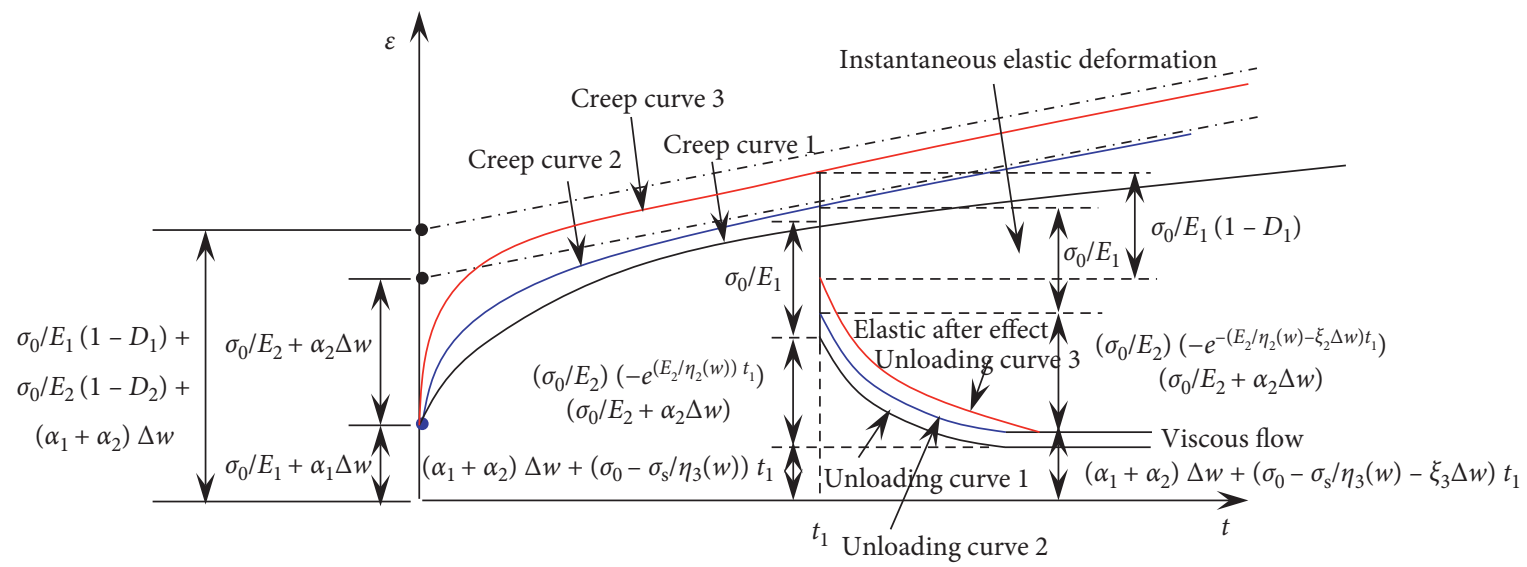

Figure 5: Model curves of creeping and unloading while $\sigma_{3 P} \geq \sigma_{S}$.

indicated two creeping states of many rocks under the effect of the humidity.

From these two curves, the effects of humidity on damage, elasticity, and viscosity are introduced into this model. It can indicate the rheological properties of rock comprehensively. With the increase of humidity, viscosity, and strength of the rock will be decreased; however, the damage and the plastic will be increased. Thus, it will cause larger creeping deformation. The model in this paper can describe the rheological properties of rock which is sensitive to the change of the humidity.

\section{Conclusions}

(1) The expansion strain is directly proportional to the mass or volume moisture content

(2) A new Nishihara model was established by introducing the effect of humidity to improve components

(3) The constitutive equations, creeping equations, and unloading equations considering the change of damage and viscosity coefficient by the effect of humidity are deduced

(4) Under the state of low stress, the model shows stable creeping; however, when it is under high stress, the model expresses unstable creeping

(5) The elastic deformation, elastic after effect, and viscous flow are occurred in the unloading curve

Problems: the model in the paper only qualitatively shows the rheological properties of the swelling rock under the effects of humidity and humidity-relative parameters theoretically. More relative experiments and field test verification should be followed up.

\section{Data Availability}

All the data used to support the findings of the study are available from the corresponding author upon requset.

\section{Conflicts of Interest}

The authors declare that there are no conflicts of interest regarding the publication of this paper.

\section{Acknowledgments}

This paper was supported by the Priority Academic Program Development of Jiangsu Higher Education Institutions and Fundamental Research Funds for the Central Universities (2017XKQY044).

\section{References}

[1] P. Xu and X.-L. Xia, "Testing study on creep characteristics of SAN-XIA granite," Chinese Jounal of Geotechnical Engineering, vol. 18, no. 4, pp. 63-67, 1996.

[2] X.-Z. Zhang, L. Wang, and D.-J. Zhang, "An experimental study on rheological characteristics of gabbro in east mountain slope of zhu-jia-bao-bao mine," Journal of Chongqing University (Natural Science Edition), vol. 22, no. 5, pp. 99-103, 1999.

[3] X.-D. Zhang, Y.-J. Li, and S.-G. Zhang, "Creep theory of soft rock and its engineering application," Chinese Journal of Rock Mechanics and Engineering, vol. 23, no. 10, pp. 1635-1639, 2004.

[4] F.-N. Jin and P. Kui-ying, "Discussion of visco-elastic models," Chinese Journal of Rock Mechanics and Engineering, vol. 14, no. 4, pp. 335-361, 1995.

[5] C. Yuan-jiang, "Research on constitutive model and intelligent identification of rock rheology," Doctoral thesis, Central South University, Changsha, China, 2003.

[6] C. Zhi-chun, M. I. A. O. Xie-xing, and M. A. O. Xian-biao, "Rock creep damage evolution equation and the fatigue damage parameter determination," Coal Science and Technology, vol. 22, no. 8, pp. 34-36, 1994.

[7] M. Xie-xing and Z.-D. Chen, "A creep damage equation for rocks," Acta Mechanica Solida Sinica, vol. 16, no. 4, pp. 343-346, 1995.

[8] Y. Yan-yi, "Underlying relationship between nonlinear rheological property of fissured rocks and damage development," Engineering Mechanics, vol. 11, no. 2, pp. 81-90, 1994. 
[9] Z. Guan, Y. Jiang, Y. Tanabashi, and H. Huang, "A new rheological model and its application in mountain tunnelling," Tunnelling and Underground Space Technology, vol. 23, no. 3, pp. 292-299, 2008.

[10] L. Zhang and S. Yang, "Unloading rheologi cal test and model research of hard rock under complex conditions," Advances in Materials Science and Engineering, vol. 2020, Article ID 3576181, 12 pages, 2020.

[11] Y. Zhao, L. Zhang, W. Wang et al., "Creep behavior of intact and cracked limestone under multi-level loading and unloading cycles," Rock Mechanics and Rock Engineering, vol. 50, no. 6, pp. 1409-1424, 2017.

[12] B. Nedjar and R. L. Roy, "An approach to the modeling of viscoelastic damage. Application to the long-term creep of gypsum rock materials," International Journal for Numerical and Analytical Methods in Geomechanics, vol. 37, no. 9, pp. 1066-1078, 2013.

[13] V. Brotóns, S. Ivorra, J. Martínez-Martínez, R. Tomás, and D. Benavente, "Estudio de la fluencia de una calcarenita: la piedra de san julián (alicante)," Materiales de Construcción, vol. 63, no. 312, pp. 581-595, 2013.

[14] P. Cao, Y. Wen, Y. Wang, Y. Haiping, and Y. Bingxiang, "Study on nonlinear damage creep constitutive model for high-stress soft rock," Environmental Earth Sciences, vol. 75, no. 10, Article ID 900, 2016.

[15] Y. Lu, D. Elsworth, and L. Wang, "A dual-scale approach to model time-dependent deformation, creep and fracturing of brittle rocks," Computers and Geotechnics, vol. 60, pp. 61-76, 2014.

[16] P. A. L. P. Firme, D. Roehl, and C. Romanel, “An assessment of the creep behaviour of Brazilian salt rocks using the multimechanism deformation model," Acta Geotechnica, vol. 11, no. 6, pp. 1445-1463, 2016.

[17] A. Fahimifar, M. Karami, and A. Fahimifar, "Modifications to an elasto-visco-plastic constitutive model for prediction of creep deformation of rock samples," Soils and foundations, vol. 55, no. 6, pp. 1364-1371, 2015.

[18] A. A. Bazhin and E. V. Murashkin, "Creep and stress relaxation in the vicinity of a micropore under the conditions of hydrostatic loading and unloading," Doklady Physics, vol. 57, no. 8, pp. 294-296, 2012.

[19] M. Xie-xing, "Large deformation analysis of surrounding rock of a tunnel in swelling rock mass based on the humidity stress field theory," Journal of China University of Mining \& Technology, vol. 24, no. 1, pp. 58-63, 1995.

[20] M. Xie-xing, A.-H. Lu, and M. A. O. Xian-biao, "Numerical simulation for roadways in swelling rock under coupling function of water and ground pressure," Journal of China University of Mining \&Technology, vol. 12, no. 2, pp. 120-125, 2002.

[21] Y Shi-lian, M. A. O. Xian-biao, and A.-H. LU, "Study of Deformation rule of swelling rock roadway under the humidity field," Journal, vol. 23, no. 4, pp. 402-405, 2006.

[22] F. Yan-qiu, Expansive Rock and Engineering, Science press, Beijing, China, 2008.

[23] X Xiao-li, "Study on the evolution of mechanical properties of granite and its micro mechanism under temperature loading," pp. 93-123, China University of Mining \&Technology, Xuzhou, China, 2008, Doctoral thesis. 